\title{
Immediate Effects of Using Insoles With Various Wedges on Plantar Pressure Measurements and Comfort Level During Marching
}

Hossein Shirvani

Baghyatoolah Medical Sciences University: Baqiyatallah University of Medical Sciences

Mohamad Hossein Ghasemi

Baghyatoolah Medical Sciences University: Baqiyatallah University of Medical Sciences

Alireza Shamsoddini ( $\nabla$ alirezaot@bmsu.ac.ir)

Baqiyatallah University of Medical Sciences https://orcid.org/0000-0001-6475-6139

Kourosh Barati

Shiraz University of Medical Sciences

Research

Keywords: Insole, Wedge, Plantar Pressure, Comfort Level

Posted Date: March 1st, 2021

DOI: https://doi.org/10.21203/rs.3.rs-258589/v1

License: (c) (i) This work is licensed under a Creative Commons Attribution 4.0 International License.

Read Full License 


\section{Abstract}

Background: Orthotic devices like insoles are widely used to improve performance. This study investigated whether immediate effects of using insoles with various wedges influence on plantar pressure measurements and comfort level during marching.

Methods: Twenty five able-bodied males participated in this study voluntarily. Repeated measures analysis of variance test with a Bonferroni correction $(\mathrm{P}<0.0014)$ was used to compare dependent variables among nine insole wedge conditions.

Results: The result of present study showed that the comfort level was non-significantly higher in the anterior and medial wedges compared to the posterior-lateral and anterior-lateral wedges during marching $(P>0.0014)$. The peak of plantar pressure and impulse in the first to third metatarsal regions decreased in the anterior, medial and anterior-medial wedges compared to the lateral and posterior-lateral wedges $(P<0.0014)$. In addition, a significant negative relation between the comfort level and plantar pressure measurements was observed in the second and third metatarsals and midfoot regions during marching $(P<0.05)$.

Conclusion: It seems that using anterior, medial and anterior-medial wedge for insole may have some advantage through increasing comfort sense and decreasing the risk of stress fracture injuries in the second and third metatarsal regions. These findings should be considered during designation of shoes or insoles for military environments.

\section{Introduction}

Military personnel often engage high physical activity leading numerous overuse injuries in the lower extremities [1]. These types of injuries resulted from completing military tasks are common and extremely costly in terms of medical treatment and rehabilitation [2]. In the literature, the most common injuries in military recruits were found in the ankle and foot areas [3], especially stress fractures of different foot regions $[4,5]$. Reports showed that $35 \%$ of the females and $41 \%$ of the males of military recruits have at least one stress fracture in the foot, with the most occurrence in the metatarsals regions [4]. If the pressure under the metatarsal head region increases, the risk of metatarsal stress fracture will be higher [6].

It was reported that high plantar pressure can produce large shocks traveling up throughout the skeletal structures and increasing the risk of stress fracture $[1,7,8]$. For example, Willems et al. (2012) [7] showed that plantar pressure in the forefoot, midfoot and medial heel increase after long-distance running, which can be related to several running injuries. Also, Weist et al. (2004) [8] stated that the fatigue can alter lower limb loading and increase the risk of overuse injuries. Hence, the assessment of plantar pressure measurements can be considered as a new method to recognize the risk of injuries $[6,7]$ and identify the effectiveness of using various modalities (like shoe and insole) [4, 9-11]. 
Previous studies showed a relation between injuries and the properties of military boots $[1,4,11]$. Adjustment of boot characteristics is a good approach in order to reduce the risk for metatarsal strain injuries and increase comfort sense $[4,11]$. Comfort, described as a subjective feeling depending on individual differences, is one of the most important aspects for shoe manufacturers [12] and the primary consideration during designation of shoes or insoles $[13,14]$. Some evidence suggested that the comfort is important for all movement-related injuries of the lower extremities [13]. On the other side, peak pressures underneath the second and third metatarsal and the heel region appear to be closely related to comfort in military boots [11].

Insole characteristics are essential for distribution of plantar pressure and perceived shoe comfort [11]. Previous research has suggested that impact forces were decreased in military boots while using an additional insole, and this can decrease the incidence of martial-related injuries [3]. Also, any change in material, designation and thicknesses of insoles can alter pressure distribution patterns and comfort level [9]. To the author knowledge, none of previous studies have investigated the effects of using various insole wedges on plantar pressure measurements and comfort level during marching. Therefore, the aim of present study was to investigate the immediate effects of using insoles with various wedges on plantar pressure measurements and comfort level during marching. The result can be useful during designation of special insoles for military environments.

\section{Methods}

\subsection{Participants}

Although the sample size of at least 24 subjects was calculated with power analysis (G*power) for statistical power 0.9 and effect size 0.25 with alpha level 0.01 , twenty-five $(n=25)$ able-bodied males (age: $23.2 \pm 3.4$ years, weight: $69.2 \pm 6.1 \mathrm{~kg}$, height: $177.4 \pm 5.4 \mathrm{~cm}$ ) participated in this study voluntarily. All subjects had no musculoskeletal disorders in the lower extremities or abnormal foot structure, ensured with the index of Williams and McClay (2000) [15].

\subsection{Description of task}

One day before the measurements, subjects had come to the laboratory and a video instruction was given them to show how to perform correct marching technique; then, they practiced this task until they were able to complete the test successfully and become familiarized with the test situation [6]. On the test day, the subjects marched straightly forward in a self-speed pace with straight legs and only with the movement of the hip joints. The peak angle for the hip joints was about $45^{\circ}( \pm 10$ error), determined with two flexible electrogoniometers (Biometrics Ltd., UK). While marching, the subjects used a coordinated arm swing so that the right arm forward with the left leg (and vice versa). The hands are cupped with the thumbs pointed down, and the arms hang straight and swing naturally. During the tests, subjects wore a standard boot which widely used in military environments [7]. For each insole condition, six successive execution of marching tests, confirmed by two professional trainers observed the task from the front and side views, were recorded [6]. 


\subsection{Designation of various insoles}

Nine type of insoles in the same material and only with various wedges were designed using SolidWorks software ver2011: Non wedge (NW), medial (MW), lateral (LW), anterior (AW), posterior (PW), posteriormedial (PM), posterior-lateral (PL), anterior-medial (AM), and anterior-lateral (AL) (Fig. 1). The angle of insole wedges was chosen according to the suggestion of Ghasemi et al. (2018) [16]. The sequence of condition was randomly completed to avoid systematic error or learning effects. Subjects rested at least 30 minutes between two consecutive conditions to avoid fatigue effects. All tests were conducted using the same boot shoes used commonly in the military environments.

\subsection{Data Analysis}

A foot scan pressure plate (RsScan International, Belgium, $40 \times 100 \mathrm{~cm}, 8192$ sensors, $253 \mathrm{~Hz}$ ) was marked along the runway to record plantar pressure measurement during the stance phase of marching [6]. The foot was automatically divided into the following ten anatomical zones by the software (Footscan1 software 7 Gait 2nd Generation, RsScan International): medial heel (HM), lateral heel ( $\mathrm{HL})$, midfoot, metatarsals from first to fifth (M1-5), and the hallux (T1) and lesser toes (T2-5). The peak pressures and impulses (absolute force time-integral) were calculated for all ten anatomical zones for each insole condition [6].

Comfort rating of each insole condition was measured six times (immediately after completing each marching task) so that the participants rated the comfort of each insole on a $150 \mathrm{~mm}$ visual analogue score (VAS), which has been designed for orthotic comfort and used in some previous studies [17]. Lower and higher scales in this index indicate to less and more comfortable sense, respectively [17]. For each condition, the average of dependent variables (including plantar pressure measurements and comfort level scores) was calculated for six marching tests.

\subsection{Ethical approval and consent to participate}

The subjects gave their written informed consent before participating in the test procedure. The study was approved by the Research Ethics Committee of Baqiyatallah University of Medical Sciences, Tehran, Iran with code: IR.BMSU.REC.1397.118

\subsection{Statistical Analysis}

For each of dependent variables, relative reliability was calculated using two-way random model of Intraclass Correlation Coefficient (ICC), with Munro's classification to describe the degree of reliability: $0.00-$ 0.25 (little); 0.26-0.49 (low); 0.50-0.69 (moderate); 0.70-0.89 (high), and 0.90-1.00 (very high) correlation [18]. Also, Shapiro-Wilk and Levene's tests were used to ensure data normality and variance homogeneity $(P<0.05)$; then, One-way Analysis of Variance (ANOVA) with repeated measures test was used to compare each dependent variable among the various insole wedges, and a Bonferroni correction was applied to prevent a violation of Type I error so that the significance difference was set at the $\mathrm{P}<$ 
0.0014 level [19]. In addition, the relation between the plantar pressure measurements and the comfort level score was measured with One-tailed Pearson's correlation coefficients test $(P<0.05)$.

\section{Results}

\subsection{Relative reliability of dependent variables}

The result of two-way random model of Intra-class Correlation Coefficient (ICC) tests showed that the relative reliability was 0.809 (high) for the comfort level and between 0.7-0.9 (high) for all plantar pressure measurements during marching task (Table 1).

Table 1

The ICC of plantar pressure variables.

\begin{tabular}{|lllll|}
\hline Variable & $\begin{array}{l}\text { Peak Pressure } \\
\text { (Left Leg) }\end{array}$ & $\begin{array}{l}\text { Peak Pressure } \\
\text { (Right Leg) }\end{array}$ & $\begin{array}{l}\text { Impulse } \\
\text { (Left Leg) }\end{array}$ & $\begin{array}{l}\text { Impulse } \\
\text { (Right Leg) }\end{array}$ \\
\hline Toe 1 & 0.764 & 0.792 & 0.788 & 0.814 \\
\hline Toe 2-5 & 0.728 & 0.746 & 0.751 & 0.779 \\
\hline Metatarsal 1 & 0.791 & 0.754 & 0.826 & 0.793 \\
\hline Metatarsal 2 & 0.813 & 0.832 & 0.803 & 0.825 \\
\hline Metatarsal 3 & 0.775 & 0.751 & 0.794 & 0.822 \\
\hline Metatarsal 4 & 0.749 & 0.794 & 0.781 & 0.743 \\
\hline Metatarsal 5 & 0.801 & 0.825 & 0.830 & 0.784 \\
\hline Midfoot & 0.757 & 0.783 & 0.783 & 0.840 \\
\hline Heel Medial & 0.780 & 0.806 & 0.816 & 0.759 \\
\hline Heel Lateral & 0.812 & 0.763 & 0.835 & 0.801 \\
\hline
\end{tabular}

\subsection{The effect of various insole wedges on the comfort level during marching}

The result of ANOVA with repeated measure test showed no significant difference among insole wedges during marching $(P>0.0014)$. However, during marching the comfort level was tended to decrease (but no significant) in the posterior-lateral wedge compared to the anterior $(P=0.002)$ and medial $(P=0.003)$ wedges, as well as in the anterior-lateral wedge compared to the anterior wedge $(P=0.007)$ (Fig. 2).

\subsection{The effect of various insole wedges on the peak plantar pressures during marching}


The result of ANOVA with repeated measure test for the peak plantar pressure showed a significant difference $(P=0.001)$ between the lateral/anterior-medial in the first metatarsal region of the right leg; medial/posterior-lateral in the second metatarsal region of the left and right leg; posterior-lateral/anteriormedial in the third metatarsal region of the left leg; lateral/posterior-medial and posterior-medial/anteriorlateral in the fifth metatarsal region of the left leg; medial/anterior-lateral and posterior-medial/anteriorlateral in the fifth metatarsal region of the right leg; posterior-lateral/anterior-medial in the lateral heel region of the left leg and right leg. No significant difference was observed in the peak pressure of other regions during marching $(P>0.0014)$ (Table 2$)$. 
Table 2

Comparison of mean (SD) of peak plantar pressures at various insole wedges during marching.

\begin{tabular}{|c|c|c|c|c|c|c|c|c|c|c|}
\hline Variable & Leg & NW & PW & AW & MW & LW & PM & PL & AM & $A L$ \\
\hline \multirow[t]{2}{*}{ Toe 1} & Left & $\begin{array}{l}165.4 \\
\pm 42.3\end{array}$ & $\begin{array}{l}176.7 \\
\pm 47.1\end{array}$ & $\begin{array}{l}158.0 \\
\pm 41.9\end{array}$ & $\begin{array}{l}151.6 \\
\pm 40.7\end{array}$ & $\begin{array}{l}168.3 \\
\pm 42.5\end{array}$ & $\begin{array}{l}162.2 \\
\pm 37.9\end{array}$ & $\begin{array}{l}171.6 \\
\pm 44.0\end{array}$ & $\begin{array}{l}158.4 \\
\pm 36.6\end{array}$ & $\begin{array}{r}176.9 \\
\pm 38.1\end{array}$ \\
\hline & Right & $\begin{array}{l}168.7 \\
\pm 45.2\end{array}$ & $\begin{array}{l}175.5 \\
\pm 50.3\end{array}$ & $\begin{array}{l}158.7 \\
\pm 43.7\end{array}$ & $\begin{array}{l}154.2 \\
\pm 44.6\end{array}$ & $\begin{array}{l}171.8 \\
\pm 48.8\end{array}$ & $\begin{array}{l}160.3 \\
\pm 38.3\end{array}$ & $\begin{array}{l}172.0 \\
\pm 47.6\end{array}$ & $\begin{array}{l}161.8 \\
\pm 39.1\end{array}$ & $\begin{array}{r}168.5 \\
\pm 36.5\end{array}$ \\
\hline \multirow[t]{2}{*}{$\begin{array}{l}\text { Toe 2- } \\
5\end{array}$} & Left & $\begin{array}{l}57.3 \\
\pm 16.2\end{array}$ & $\begin{array}{l}58.9 \\
\pm 17.7\end{array}$ & $\begin{array}{l}54.1 \\
\pm 18.3\end{array}$ & $\begin{array}{l}65.3 \\
\pm 16.4\end{array}$ & $\begin{array}{l}58.5 \\
\pm 16.9\end{array}$ & $\begin{array}{l}64.2 \\
\pm 15.1\end{array}$ & $\begin{array}{l}55.9 \\
\pm 16.6\end{array}$ & $\begin{array}{l}60.0 \\
\pm 16.0\end{array}$ & $\begin{array}{l}63.7 \\
\pm 17.9\end{array}$ \\
\hline & Right & $\begin{array}{l}60.9 \\
\pm 18.0\end{array}$ & $\begin{array}{l}58.3 \\
\pm 16.2\end{array}$ & $\begin{array}{l}57.5 \\
\pm 14.9\end{array}$ & $\begin{array}{l}69.2 \\
\pm 18.3\end{array}$ & $\begin{array}{l}54.7 \\
\pm 17.7\end{array}$ & $\begin{array}{l}65.6 \\
\pm 16.8\end{array}$ & $\begin{array}{l}57.4 \\
\pm 15.2\end{array}$ & $\begin{array}{l}64.5 \\
\pm 15.9\end{array}$ & $\begin{array}{l}60.8 \\
\pm 18.0\end{array}$ \\
\hline \multirow[t]{2}{*}{ Meta 1} & Left & $\begin{array}{l}208.3 \\
\pm 47.9\end{array}$ & $\begin{array}{l}223.2 \\
\pm 41.8\end{array}$ & $\begin{array}{l}213.7 \\
\pm 46.5\end{array}$ & $\begin{array}{l}207.9 \\
\pm 48.1\end{array}$ & $\begin{array}{l}228.1 \\
\pm 44.9\end{array}$ & $\begin{array}{l}214.4 \\
\pm 50.2\end{array}$ & $\begin{array}{l}232.6 \\
\pm 46.6\end{array}$ & $\begin{array}{l}203.1 \\
\pm 50.3\end{array}$ & $\begin{array}{l}217.8 \\
\pm 41.4\end{array}$ \\
\hline & Right & $\begin{array}{l}214.5 \\
\pm 51.3\end{array}$ & $\begin{array}{l}228.9 \\
\pm 44.5\end{array}$ & $\begin{array}{l}203.7 \\
\pm 53.0\end{array}$ & $\begin{array}{l}205.8 \\
\pm 47.4\end{array}$ & $\begin{array}{l}237.2 \\
\pm \\
52.1^{\#}\end{array}$ & $\begin{array}{l}212.5 \\
\pm 51.7\end{array}$ & $\begin{array}{l}220.7 \\
\pm 47.9\end{array}$ & $\begin{array}{l}199.8 \\
\pm 45.6\end{array}$ & $\begin{array}{l}211.3 \\
\pm 48.2\end{array}$ \\
\hline \multirow[t]{2}{*}{ Meta 2} & Left & $\begin{array}{l}242.4 \\
\pm 42.7\end{array}$ & $\begin{array}{l}240.8 \\
\pm 37.0\end{array}$ & $\begin{array}{l}232.1 \\
\pm 38.3\end{array}$ & $\begin{array}{l}227.0 \\
\pm \\
43.6^{*}\end{array}$ & $\begin{array}{l}246.5 \\
\pm 40.4\end{array}$ & $\begin{array}{l}250.4 \\
\pm 45.3\end{array}$ & $\begin{array}{l}256.3 \\
\pm 46.5\end{array}$ & $\begin{array}{l}245.5 \\
\pm 49.3\end{array}$ & $\begin{array}{l}253.8 \\
\pm 41.5\end{array}$ \\
\hline & Right & $\begin{array}{l}236.2 \\
\pm 50.3\end{array}$ & $\begin{array}{l}232.5 \\
\pm 40.9\end{array}$ & $\begin{array}{l}228.8 \\
\pm 47.5\end{array}$ & $\begin{array}{l}219.7 \\
\pm \\
49.2^{\star}\end{array}$ & $\begin{array}{l}252.2 \\
\pm 41.7\end{array}$ & $\begin{array}{l}247.8 \\
\pm 48.8\end{array}$ & $\begin{array}{l}259.1 \\
\pm 51.1\end{array}$ & $\begin{array}{l}239.8 \\
\pm 42.6\end{array}$ & $\begin{array}{l}246.4 \\
\pm 43.4\end{array}$ \\
\hline \multirow[t]{2}{*}{ Meta 3} & Left & $\begin{array}{l}218.8 \\
\pm 38.0\end{array}$ & $\begin{array}{l}224.4 \\
\pm 40.4\end{array}$ & $\begin{array}{l}208.1 \\
\pm 35.9\end{array}$ & $\begin{array}{l}222.2 \\
\pm 47.4\end{array}$ & $\begin{array}{l}234.9 \\
\pm 43.6\end{array}$ & $\begin{array}{l}229.0 \\
\pm 40.9\end{array}$ & $\begin{array}{l}233.6 \\
\pm \\
45.0^{\#}\end{array}$ & $\begin{array}{l}205.0 \\
\pm 40.1\end{array}$ & $\begin{array}{l}218.7 \\
\pm 37.0\end{array}$ \\
\hline & Right & $\begin{array}{l}213.7 \\
\pm 41.7\end{array}$ & $\begin{array}{l}228.8 \\
\pm 47.4\end{array}$ & $\begin{array}{l}209.0 \\
\pm 42.1\end{array}$ & $\begin{array}{l}220.8 \\
\pm 39.3\end{array}$ & $\begin{array}{l}227.7 \\
\pm 44.6\end{array}$ & $\begin{array}{l}233.9 \\
\pm 45.5\end{array}$ & $\begin{array}{l}236.6 \\
\pm 39.2\end{array}$ & $\begin{array}{l}208.2 \\
\pm 46.3\end{array}$ & $\begin{array}{l}213.0 \\
\pm 36.5\end{array}$ \\
\hline \multirow[t]{2}{*}{ Meta 4} & Left & $\begin{array}{l}143.4 \\
\pm 39.2\end{array}$ & $\begin{array}{l}156.1 \\
\pm 35.5\end{array}$ & $\begin{array}{l}148.3 \\
\pm 34.1\end{array}$ & $\begin{array}{l}160.9 \\
\pm 39.1\end{array}$ & $\begin{array}{l}145.2 \\
\pm 42.4\end{array}$ & $\begin{array}{l}162.6 \\
\pm 33.6\end{array}$ & $\begin{array}{l}150.7 \\
\pm 35.2\end{array}$ & $\begin{array}{l}150.0 \\
\pm 38.8\end{array}$ & $\begin{array}{l}140.3 \\
\pm 40.6\end{array}$ \\
\hline & Right & $\begin{array}{l}149.7 \\
\pm 42.3\end{array}$ & $\begin{array}{l}156.4 \\
\pm 39.2\end{array}$ & $\begin{array}{l}147.1 \\
\pm 35.7\end{array}$ & $\begin{array}{l}154.8 \\
\pm 44.8\end{array}$ & $\begin{array}{l}149.2 \\
\pm 40.6\end{array}$ & $\begin{array}{l}159.3 \\
\pm 39.4\end{array}$ & $\begin{array}{l}147.4 \\
\pm 37.1\end{array}$ & $\begin{array}{l}146.8 \\
\pm 44.1\end{array}$ & $\begin{array}{l}140.5 \\
\pm 42.5\end{array}$ \\
\hline \multirow[t]{2}{*}{ Meta 5} & Left & $\begin{array}{l}96.2 \\
\pm 28.7\end{array}$ & $\begin{array}{l}102.0 \\
\pm 22.1\end{array}$ & $\begin{array}{l}99.4 \\
\pm 21.2\end{array}$ & $\begin{array}{l}111.9 \\
\pm \\
26.6^{\&}\end{array}$ & $\begin{array}{l}94.1 \\
\pm 22.7\end{array}$ & $\begin{array}{l}114.4 \\
\pm 22.2\end{array}$ & $\begin{array}{l}100.4 \\
\pm 27.3\end{array}$ & $\begin{array}{l}102.9 \\
\pm 24.8\end{array}$ & $\begin{array}{l}92.5 \\
\pm \\
26.0^{\&}\end{array}$ \\
\hline & Right & $\begin{array}{l}94.1 \\
\pm 25.0\end{array}$ & $\begin{array}{l}107.4 \\
\pm 27.3\end{array}$ & $\begin{array}{l}97.7 \\
\pm 26.6\end{array}$ & $\begin{array}{l}113.8 \\
\pm \\
23.1^{9}\end{array}$ & $\begin{array}{l}95.4 \\
\pm 23.9\end{array}$ & $\begin{array}{l}115.3 \\
\pm \\
24.0\end{array}$ & $\begin{array}{l}102.2 \\
\pm 27.9\end{array}$ & $\begin{array}{l}106.1 \\
\pm 24.7\end{array}$ & $\begin{array}{l}92.4 \\
\pm 22.9\end{array}$ \\
\hline
\end{tabular}

The unit of Peak Pressure values is $\mathrm{N} / \mathrm{cm} 2$; The signs $\&, *$, \# and 9 indicate to significant difference between the PM, PL, AM and AL conditions and the targeted condition, respectively $(P<0.001)$. 


\begin{tabular}{|c|c|c|c|c|c|c|c|c|c|c|}
\hline Variable & Leg & NW & PW & AW & MW & LW & PM & PL & AM & AL \\
\hline \multirow[t]{2}{*}{ Midfoot } & Left & $\begin{array}{l}65.5 \\
\pm 12.3\end{array}$ & $\begin{array}{l}68.8 \\
\pm 12.5\end{array}$ & $\begin{array}{l}62.1 \\
\pm 16.4\end{array}$ & $\begin{array}{l}64.1 \\
\pm 12.6\end{array}$ & $\begin{array}{l}68.8 \\
\pm 14.0\end{array}$ & $\begin{array}{l}66.9 \\
\pm 15.2\end{array}$ & $\begin{array}{l}70.6 \\
\pm 14.3\end{array}$ & $\begin{array}{l}65.3 \\
\pm 14.7\end{array}$ & $\begin{array}{l}67.6 \\
\pm 12.7\end{array}$ \\
\hline & Right & $\begin{array}{l}63.1 \\
\pm 14.1\end{array}$ & $\begin{array}{l}65.1 \\
\pm 15.2\end{array}$ & $\begin{array}{l}68.3 \\
\pm 13.3\end{array}$ & $\begin{array}{l}66.9 \\
\pm 15.2\end{array}$ & $\begin{array}{l}65.1 \\
\pm 15.8\end{array}$ & $\begin{array}{l}63.5 \\
\pm 13.4\end{array}$ & $\begin{array}{l}65.9 \\
\pm 13.0\end{array}$ & $\begin{array}{l}64.6 \\
\pm 16.8\end{array}$ & $\begin{array}{l}62.9 \\
\pm 15.6\end{array}$ \\
\hline \multirow[t]{2}{*}{$\begin{array}{l}\text { Heel } \\
\text { Medial }\end{array}$} & Left & $\begin{array}{l}154.3 \\
\pm 38.3\end{array}$ & $\begin{array}{l}152.4 \\
\pm 38.1\end{array}$ & $\begin{array}{l}166.2 \\
\pm 41.2\end{array}$ & $\begin{array}{l}156.3 \\
\pm 40.5\end{array}$ & $\begin{array}{l}148.8 \\
\pm 32.8\end{array}$ & $\begin{array}{l}147.3 \\
\pm 35.4\end{array}$ & $\begin{array}{l}150.1 \\
\pm 41.6\end{array}$ & $\begin{array}{l}152.4 \\
\pm 41.4\end{array}$ & $\begin{array}{l}169.7 \\
\pm 33.4\end{array}$ \\
\hline & Right & $\begin{array}{l}161.7 \\
\pm 33.5\end{array}$ & $\begin{array}{l}158.9 \\
\pm 41.6\end{array}$ & $\begin{array}{l}161.3 \\
\pm 33.5\end{array}$ & $\begin{array}{l}152.5 \\
\pm 36.1\end{array}$ & $\begin{array}{r}150.9 \\
\pm 39.2\end{array}$ & $\begin{array}{l}159.1 \\
\pm 42.1\end{array}$ & $\begin{array}{l}157.2 \\
\pm 40.9\end{array}$ & $\begin{array}{l}160.4 \\
\pm 36.9\end{array}$ & $\begin{array}{l}166.7 \\
\pm 41.2\end{array}$ \\
\hline \multirow[t]{2}{*}{$\begin{array}{l}\text { Heel } \\
\text { Lateral }\end{array}$} & Left & $\begin{array}{l}145.1 \\
\pm 36.2\end{array}$ & $\begin{array}{l}139.2 \\
\pm 30.6\end{array}$ & $\begin{array}{l}157.3 \\
\pm 36.6\end{array}$ & $\begin{array}{l}151.5 \\
\pm 32.0\end{array}$ & $\begin{array}{l}148.7 \\
\pm 36.8\end{array}$ & $\begin{array}{l}149.7 \\
\pm 35.9\end{array}$ & $\begin{array}{l}137.8 \\
\pm 36.0\end{array}$ & $\begin{array}{l}163.0 \\
\pm 33.3\end{array}$ & $\begin{array}{r}150.3 \\
\pm 38.4\end{array}$ \\
\hline & Right & $\begin{array}{l}151.8 \\
\pm 39.1\end{array}$ & $\begin{array}{l}145.7 \\
\pm 34.1\end{array}$ & $\begin{array}{l}151.6 \\
\pm 37.6\end{array}$ & $\begin{array}{l}149.8 \\
\pm 38.3\end{array}$ & $\begin{array}{l}143.2 \\
\pm 31.2\end{array}$ & $\begin{array}{l}159.2 \\
\pm 41.4\end{array}$ & $\begin{array}{l}134.3 \\
\pm \\
31.2^{\#}\end{array}$ & $\begin{array}{l}167.6 \\
\pm 37.1\end{array}$ & $\begin{array}{r}157.8 \\
\pm 32.4\end{array}$ \\
\hline
\end{tabular}

\subsection{The effect of various insole wedges on the impulses during marching}

The result of ANOVA with repeated measure test for the impulse values showed a significant difference $(P$ $=0.001$ ) between the posterior-lateral/anterior-medial in the first metatarsal region of the left and right leg; medial/posterior-lateral in the first metatarsal region of the right leg; anterior/posterior-lateral and posterior-lateral/anterior-medial in the second metatarsal region of the left leg; non-wedge/posteriormedial in the fifth metatarsal region of the left leg; medial/lateral and lateral/posterior-medial in the fifth metatarsal region of the right leg; posterior-medial/anterior-lateral in the medial heel region of the right leg. No significant difference was observed in the impulse of other regions during marching $(P>0.0014)$ (Table 3). 
Table 3

Comparison of mean (SD) of impulses at various insole wedges during marching.

\begin{tabular}{|c|c|c|c|c|c|c|c|c|c|c|}
\hline Variable & Leg & NW & PW & AW & MW & LW & PM & $\mathrm{PL}$ & AM & $\mathrm{AL}$ \\
\hline \multirow[t]{2}{*}{ Toe 1} & Left & $\begin{array}{l}26.9 \\
\pm 6.1\end{array}$ & $\begin{array}{l}25.3 \\
\pm 5.8\end{array}$ & $\begin{array}{l}27.3 \\
\pm 6.4\end{array}$ & $\begin{array}{l}24.0 \pm \\
6.6\end{array}$ & $\begin{array}{l}26.8 \\
\pm 6.8\end{array}$ & $\begin{array}{l}24.9 \pm \\
5.9\end{array}$ & $\begin{array}{l}28.6 \\
\pm 6.4\end{array}$ & $\begin{array}{l}25.4 \\
\pm 6.3\end{array}$ & $\begin{array}{l}27.7 \pm \\
6.1\end{array}$ \\
\hline & Right & $\begin{array}{l}28.4 \\
\pm 6.5\end{array}$ & $\begin{array}{l}24.6 \\
\pm 6.7\end{array}$ & $\begin{array}{l}27.8 \\
\pm 5.9\end{array}$ & $\begin{array}{l}25.5 \pm \\
6.3\end{array}$ & $\begin{array}{l}25.3 \\
\pm 6.2\end{array}$ & $\begin{array}{l}25.9 \pm \\
5.7\end{array}$ & $\begin{array}{l}24.5 \\
\pm 5.4\end{array}$ & $\begin{array}{l}26.6 \\
\pm 6.6\end{array}$ & $\begin{array}{l}28.6 \pm \\
5.8\end{array}$ \\
\hline \multirow[t]{2}{*}{$\begin{array}{l}\text { Toe 2- } \\
5\end{array}$} & Left & $\begin{array}{l}12.7 \\
\pm 3.8\end{array}$ & $\begin{array}{l}11.5 \\
\pm 4.2\end{array}$ & $\begin{array}{l}13.3 \\
\pm 3.5\end{array}$ & $\begin{array}{l}13.5 \pm \\
3.4\end{array}$ & $\begin{array}{l}12.3 \\
\pm 4.1\end{array}$ & $\begin{array}{l}13.3 \pm \\
3.4\end{array}$ & $\begin{array}{l}12.5 \\
\pm 3.6\end{array}$ & $\begin{array}{l}13.1 \\
\pm 3.4\end{array}$ & $\begin{array}{l}13.3 \pm \\
4.0\end{array}$ \\
\hline & Right & $\begin{array}{l}12.4 \\
\pm 3.6\end{array}$ & $\begin{array}{l}12.3 \\
\pm 3.7\end{array}$ & $\begin{array}{l}13.5 \\
\pm 3.4\end{array}$ & $\begin{array}{l}12.9 \pm \\
4.0\end{array}$ & $\begin{array}{l}12.7 \\
\pm 3.6\end{array}$ & $\begin{array}{l}14.0 \pm \\
3.8\end{array}$ & $\begin{array}{l}11.9 \\
\pm 3.3\end{array}$ & $\begin{array}{l}12.8 \\
\pm 4.1\end{array}$ & $\begin{array}{l}13.9 \pm \\
3.8\end{array}$ \\
\hline \multirow[t]{2}{*}{ Meta 1} & Left & $\begin{array}{l}32.4 \\
\pm 7.2\end{array}$ & $\begin{array}{l}35.9 \\
\pm 7.0\end{array}$ & $\begin{array}{l}33.4 \\
\pm 6.9\end{array}$ & $\begin{array}{l}31.7 \pm \\
6.9\end{array}$ & $\begin{array}{l}36.0 \\
\pm 6.7\end{array}$ & $\begin{array}{l}35.6 \pm \\
7.5\end{array}$ & $\begin{array}{l}37.5 \\
\pm 6.4\end{array}$ & $\begin{array}{l}32.0 \\
\pm 6.3^{*}\end{array}$ & $\begin{array}{l}34.8 \pm \\
6.2\end{array}$ \\
\hline & Right & $\begin{array}{l}33.6 \\
\pm 6.3\end{array}$ & $\begin{array}{l}34.4 \\
\pm 7.2\end{array}$ & $\begin{array}{l}35.5 \\
\pm 6.4\end{array}$ & $\begin{array}{l}31.4 \pm \\
7.4^{*}\end{array}$ & $\begin{array}{l}36.2 \\
\pm 6.6\end{array}$ & $\begin{array}{l}33.8 \pm \\
7.6\end{array}$ & $\begin{array}{l}38.1 \\
\pm 6.2\end{array}$ & $\begin{array}{l}31.5 \\
\pm 6.9^{*}\end{array}$ & $\begin{array}{l}34.2 \pm \\
6.7\end{array}$ \\
\hline \multirow[t]{2}{*}{ Meta 2} & Left & $\begin{array}{l}39.1 \\
\pm 7.4\end{array}$ & $\begin{array}{l}38.4 \\
\pm 7.0\end{array}$ & $\begin{array}{l}36.9 \\
\pm 6.4^{*}\end{array}$ & $\begin{array}{l}37.4 \pm \\
7.1\end{array}$ & $\begin{array}{l}38.9 \\
\pm 6.3\end{array}$ & $\begin{array}{l}39.1 \pm \\
6.7\end{array}$ & $\begin{array}{l}42.4 \\
\pm 7.2\end{array}$ & $\begin{array}{l}36.8 \\
\pm 6.0^{*}\end{array}$ & $\begin{array}{l}40.5 \pm \\
6.5\end{array}$ \\
\hline & Right & $\begin{array}{l}37.9 \\
\pm 6.3\end{array}$ & $\begin{array}{l}37.3 \\
\pm 6.4\end{array}$ & $\begin{array}{l}38.6 \\
\pm 6.9\end{array}$ & $\begin{array}{l}36.9 \pm \\
6.4\end{array}$ & $\begin{array}{l}39.3 \\
\pm 7.0\end{array}$ & $\begin{array}{l}38.8 \pm \\
7.5\end{array}$ & $\begin{array}{l}41.2 \\
\pm 6.8\end{array}$ & $\begin{array}{l}37.2 \\
\pm 7.2\end{array}$ & $\begin{array}{l}39.8 \pm \\
6.9\end{array}$ \\
\hline \multirow[t]{2}{*}{ Meta 3} & Left & $\begin{array}{l}31.9 \\
\pm 6.6\end{array}$ & $\begin{array}{l}31.8 \\
\pm 6.3\end{array}$ & $\begin{array}{l}29.5 \\
\pm 6.7\end{array}$ & $\begin{array}{l}30.5 \pm \\
7.1\end{array}$ & $\begin{array}{l}32.6 \\
\pm 6.1\end{array}$ & $\begin{array}{l}31.4 \pm \\
6.5\end{array}$ & $\begin{array}{l}31.7 \\
\pm 7.0\end{array}$ & $\begin{array}{l}29.4 \\
\pm 6.7\end{array}$ & $\begin{array}{l}32.0 \pm \\
6.3\end{array}$ \\
\hline & Right & $\begin{array}{l}33.2 \\
\pm 6.1\end{array}$ & $\begin{array}{l}32.5 \\
\pm 7.4\end{array}$ & $\begin{array}{l}27.8 \\
\pm 6.9\end{array}$ & $\begin{array}{l}31.3 \pm \\
7.7\end{array}$ & $\begin{array}{l}32.7 \\
\pm 7.0\end{array}$ & $\begin{array}{l}32.4 \pm \\
6.9\end{array}$ & $\begin{array}{l}32.1 \\
\pm 6.8\end{array}$ & $\begin{array}{l}31.5 \\
\pm 7.3\end{array}$ & $\begin{array}{l}30.7 \pm \\
6.8\end{array}$ \\
\hline \multirow[t]{2}{*}{ Meta 4} & Left & $\begin{array}{l}26.6 \\
\pm 7.1\end{array}$ & $\begin{array}{l}23.1 \\
\pm 6.2\end{array}$ & $\begin{array}{l}25.0 \\
\pm 6.6\end{array}$ & $\begin{array}{l}24.1 \pm \\
6.8\end{array}$ & $\begin{array}{l}23.7 \\
\pm 6.1\end{array}$ & $\begin{array}{l}26.3 \pm \\
6.2\end{array}$ & $\begin{array}{l}24.8 \\
\pm 5.9\end{array}$ & $\begin{array}{l}24.6 \\
\pm 7.0\end{array}$ & $\begin{array}{l}22.4 \pm \\
6.1\end{array}$ \\
\hline & Right & $\begin{array}{l}27.1 \\
\pm 6.4\end{array}$ & $\begin{array}{l}22.4 \\
\pm 6.6\end{array}$ & $\begin{array}{l}26.1 \\
\pm 7.2\end{array}$ & $\begin{array}{l}25.2 \pm \\
6.6\end{array}$ & $\begin{array}{l}24.2 \\
\pm 6.8\end{array}$ & $\begin{array}{l}23.9 \pm \\
7.0\end{array}$ & $\begin{array}{l}23.7 \\
\pm 6.3\end{array}$ & $\begin{array}{l}25.9 \\
\pm 7.2\end{array}$ & $\begin{array}{l}24.7 \pm \\
6.5\end{array}$ \\
\hline \multirow[t]{2}{*}{ Meta 5} & Left & $\begin{array}{l}11.9 \\
\pm 3.4^{\&}\end{array}$ & $\begin{array}{l}13.3 \\
\pm 3.5\end{array}$ & $\begin{array}{r}12.9 \\
\pm 3.6\end{array}$ & $\begin{array}{l}14.3 \pm \\
3.9\end{array}$ & $\begin{array}{r}12.2 \\
\pm 3.6\end{array}$ & $\begin{array}{l}15.2 \pm \\
3.2\end{array}$ & $\begin{array}{l}13.4 \\
\pm 4.1\end{array}$ & $\begin{array}{l}14.1 \\
\pm 3.7\end{array}$ & $\begin{array}{l}12.1 \pm \\
3.2\end{array}$ \\
\hline & Right & $\begin{array}{l}12.6 \\
\pm 3.2\end{array}$ & $\begin{array}{l}14.0 \\
\pm 3.7\end{array}$ & $\begin{array}{l}13.4 \\
\pm 3.9\end{array}$ & $\begin{array}{l}14.7 \pm \\
3.5 @\end{array}$ & $\begin{array}{l}11.6 \\
\pm 3.1\end{array}$ & $\begin{array}{l}15.0 \pm \\
3.5 @\end{array}$ & $\begin{array}{l}14.2 \\
\pm 3.3\end{array}$ & $\begin{array}{l}13.7 \\
\pm 4.0\end{array}$ & $\begin{array}{l}13.4 \pm \\
3.9\end{array}$ \\
\hline \multirow[t]{2}{*}{ Midfoot } & Left & $\begin{array}{l}31.4 \\
\pm 6.2\end{array}$ & $\begin{array}{l}32.2 \\
\pm 6.6\end{array}$ & $\begin{array}{l}29.0 \\
\pm 6.8\end{array}$ & $\begin{array}{l}29.9 \pm \\
7.0\end{array}$ & $\begin{array}{l}27.5 \\
\pm 6.2\end{array}$ & $\begin{array}{l}30.9 \pm \\
5.9\end{array}$ & $\begin{array}{l}31.3 \\
\pm 6.8\end{array}$ & $\begin{array}{l}28.8 \\
\pm 7.1\end{array}$ & $\begin{array}{l}30.5 \pm \\
6.3\end{array}$ \\
\hline & Right & $\begin{array}{l}29.5 \\
\pm 7.0\end{array}$ & $\begin{array}{l}31.5 \\
\pm 6.9\end{array}$ & $\begin{array}{l}26.7 \\
\pm 6.5\end{array}$ & $\begin{array}{l}31.0 \pm \\
6.4\end{array}$ & $\begin{array}{l}29.8 \\
\pm 5.8\end{array}$ & $\begin{array}{l}27.4 \pm \\
6.7\end{array}$ & $\begin{array}{l}32.5 \\
\pm 6.4\end{array}$ & $\begin{array}{l}28.1 \\
\pm 6.6\end{array}$ & $\begin{array}{l}31.1 \pm \\
7.2\end{array}$ \\
\hline
\end{tabular}

The unit of Impulse is values N.s/ $\mathrm{cm}^{2}$.

The signs @, \& and * indicate to significant difference between the LW, PM and PL conditions and the targeted condition, respectively $(P<0.001)$. 


\begin{tabular}{|c|c|c|c|c|c|c|c|c|c|c|}
\hline Variable & Leg & NW & PW & AW & MW & LW & PM & PL & AM & AL \\
\hline \multirow[t]{2}{*}{$\begin{array}{l}\text { Heel } \\
\text { Medial }\end{array}$} & Left & $\begin{array}{l}20.3 \\
\pm 6.3\end{array}$ & $\begin{array}{l}19.3 \\
\pm 6.1\end{array}$ & $\begin{array}{l}23.7 \\
\pm 5.7\end{array}$ & $\begin{array}{l}20.8 \pm \\
6.2\end{array}$ & $\begin{array}{r}19.5 \\
\pm 5.6\end{array}$ & $\begin{array}{l}18.9 \pm \\
5.4\end{array}$ & $\begin{array}{l}20.4 \\
\pm 5.7\end{array}$ & $\begin{array}{l}19.4 \\
\pm 5.8\end{array}$ & $\begin{array}{l}22.6 \pm \\
5.2\end{array}$ \\
\hline & Right & $\begin{array}{l}18.5 \\
\pm 6.1\end{array}$ & $\begin{array}{l}21.0 \\
\pm 6.5\end{array}$ & $\begin{array}{l}21.7 \\
\pm 6.1\end{array}$ & $\begin{array}{l}21.9 \pm \\
6.0\end{array}$ & $\begin{array}{l}19.3 \\
\pm 6.7\end{array}$ & $\begin{array}{l}18.7 \pm \\
6.0\end{array}$ & $\begin{array}{l}19.6 \\
\pm 5.1\end{array}$ & $\begin{array}{l}21.2 \\
\pm 6.3\end{array}$ & $\begin{array}{l}23.1 \pm \\
5.6^{\&}\end{array}$ \\
\hline \multirow[t]{2}{*}{$\begin{array}{l}\text { Heel } \\
\text { Lateral }\end{array}$} & Left & $\begin{array}{l}14.8 \\
\pm 4.0\end{array}$ & $\begin{array}{r}16.5 \\
\pm 4.2\end{array}$ & $\begin{array}{l}17.2 \\
\pm 5.0\end{array}$ & $\begin{array}{l}15.3 \pm \\
4.7\end{array}$ & $\begin{array}{l}16.0 \\
\pm 4.5\end{array}$ & $\begin{array}{l}17.3 \pm \\
4.4\end{array}$ & $\begin{array}{l}15.1 \\
\pm 4.8\end{array}$ & $\begin{array}{l}17.2 \\
\pm 4.2\end{array}$ & $\begin{array}{l}16.1 \pm \\
4.6\end{array}$ \\
\hline & Right & $\begin{array}{l}15.5 \\
\pm 4.7\end{array}$ & $\begin{array}{l}14.9 \\
\pm 4.8\end{array}$ & $\begin{array}{l}16.1 \\
\pm 4.6\end{array}$ & $\begin{array}{l}16.3 \pm \\
4.3\end{array}$ & $\begin{array}{l}15.8 \\
\pm 4.1\end{array}$ & $\begin{array}{l}17.7 \pm \\
4.9\end{array}$ & $\begin{array}{l}14.9 \\
\pm 5.0\end{array}$ & $\begin{array}{l}16.2 \\
\pm 4.8\end{array}$ & $\begin{array}{l}15.8 \pm \\
4.1\end{array}$ \\
\hline \multicolumn{11}{|c|}{ The unit of Impulse is values $\mathrm{N} . \mathrm{s} / \mathrm{cm}^{2}$. } \\
\hline
\end{tabular}

\subsection{The relation between comfort level and plantar pressure measurements during marching}

The result of One-tailed Pearson's correlation coefficients tests showed a significant negative relation during marching between the comfort level and plantar pressure measurements in these regions: the second metatarsal $(P=0.001)$, the third metatarsal $(P=0.001)$ and the midfoot $(P=0.001$ for the peak pressure of the left and right leg and $P=0.033$ for the impulse of the right leg) (Table 4). 
Table 4

Pearson value of correlation test between comfort level and plantar pressure measurements.

\begin{tabular}{|lllll|}
\hline Variable & $\begin{array}{l}\text { Peak Pressure } \\
\text { (Left Leg) }\end{array}$ & $\begin{array}{l}\text { Peak Pressure } \\
\text { (Right Leg) }\end{array}$ & $\begin{array}{l}\text { Impulse } \\
\text { (Left Leg) }\end{array}$ & $\begin{array}{l}\text { Impulse } \\
\text { (Right Leg) }\end{array}$ \\
\hline Toe 1 & -0.09 & -0.003 & -0.055 & -0.023 \\
\hline Toe 2-5 & -0.065 & -0.044 & -0.019 & -0.079 \\
\hline Metatarsal 1 & -0.108 & -0.112 & 0.056 & 0.038 \\
\hline Metatarsal 2 & $-0.459^{* \star}$ & $-0.424^{* \star}$ & $-0.308^{* \star}$ & $-0.275^{* \star}$ \\
\hline Metatarsal 3 & $-0.305^{* *}$ & $-0.302^{* \star}$ & $-0.280^{* \star}$ & $-0.226^{* \star}$ \\
\hline Metatarsal 4 & -0.005 & -0.077 & 0.043 & -0.029 \\
\hline Metatarsal 5 & -0.075 & -0.026 & -0.007 & 0.040 \\
\hline Midfoot & $-0.355^{* *}$ & $-0.319^{* \star}$ & -0.124 & $-0.142^{*}$ \\
\hline Heel Medial & 0.033 & -0.068 & 0.058 & 0.106 \\
\hline Heel Lateral & -0.069 & -0.050 & -0.060 & -0.078 \\
\hline The * and ** signs indicate to the significant correlation at $P<0.05$ and $P<0.01$, respectively. \\
\hline
\end{tabular}

\section{Discussion}

The aim of present study was to investigate the immediate effects of using insoles with various wedges on plantar pressure measurements and comfort level during marching. Our results showed high relative reliability for the comfort level and all plantar pressure measurements during marching task (ICC >0.7).

The result of present study showed that the comfort level was non-significantly higher in the anterior and medial wedges compared to the posterior-lateral and anterior-lateral wedges during marching. Comfort is an important property for military boots [11] and the primary consideration during designation of shoes or insoles $[13,14]$. In this area, insole characteristics have been introduced as an important factor influencing on comfort sense [11], and some investigators tried to find a way to increase the comfortability of military boots [3, 13,20]. For example, Paisis et al. (2013) [3] showed that using two types of insoles in the military boots increase the comfort sense non-significantly; they concluded that changes in designation of military boots, such as softer leather and wider shoe lasts, may have some benefits. In a study on 106 soldiers, Muendermann et al. (2001) [20] compared six different insoles (different with respect to shape and material) and the subjects used their most comfortable insole for the next 4 months. Their results showed that using comfortable insole can decrease lower-extremity injuries [20]. In addition, Yung-Hui and WeiHsien (2005) [10] showed that using high-heel shoes significantly decrease and using some inserts (like arch support, heel cup and metatarsal pad) increase footwear comfort. As some evidence suggested that 
the comfort is important for all movement-related injuries of the lower extremities $[13,20]$ our results may indicate that the anterior and medial wedges may have some benefits during completing marching task.

The result of present study showed significant (or non-significant) differences among the insole wedges for the peak of plantar pressure and impulse mainly in the first, second, third and fifth metatarsals and medial and lateral heel regions. Generally, the peak of plantar pressure and impulse in the first to third metatarsal regions decreased in the anterior, medial and anterior-medial wedges compared to the lateral and posterior-lateral wedges. In the heel regions, the difference in the peak of plantar pressure and impulse was observed between posterior-lateral/anterior-medial wedges and posterior-medial/anterior-lateral wedges. These findings are in accordance with the study of some researchers who tried to find a way to decrease plantar pressures in these regions for military populations [1-5]. For example, Windle et al. (1999) [5] applied four different insoles (based on material characteristics) in military boots and showed significant difference in the peak plantar pressures among the insoles during running and marching. House et al. (2004) [2] observed that two thicknesses of insole ( $3 \mathrm{~mm}$ and $6 \mathrm{~mm}$ ) significantly decreased the plantar peak pressures compared to no-insole condition. Yung-Hui and Wei-Hsien (2005) [10] showed that a heel cup insert for high-heeled shoes effectively decreased the heel pressure and impact force and an arch support insert decreased the medial forefoot pressure. Also, Paisis et al. (2013) [3] stated that for some individuals, using insoles in military boots may have an important benefit by decreasing ground reaction forces. In addition, Muniz and Bini (2017) [1] compared three different military boots and showed that the thicker midsole boot was more effective in decreasing impact of ground reaction forces during walking. However, none of previous studies have tried to find optimal insole designation for military environments. Given the above, it seems that using anterior, medial and anterior-medial wedge for insole may have some advantage through decreasing the risk of stress fracture injuries in the second and third metatarsal regions, having high incidence rate in military recruits [4]. These findings should be considered during designation of shoes or insoles for military environments.

The result of present study showed a significant negative relation between the comfort level and plantar pressure measurements in the second and third metatarsals and midfoot regions during marching. These findings are in accordance with the study of Lange et al. (2009), Paisis et al. (2013), Xu et al. (2019) and Yang et al. (2019) $[3,11,14,21]$ who showed negative correlation between the comfort level and plantar pressures (or ground reaction forces). This relation between subjective (comfort sense) and objective (plantar pressure measurements) data can be useful during designation assessment of various insoles. It seems that the assessment of comfort may be useful and used instead of plantar pressure measurements when specialized tools (like foot scan) is not existed. However, future studies are needed to assess other biomechanical aspects.

\section{Conclusions}

The result of present study showed that the comfort level was non-significantly higher in the anterior and medial wedges compared to the posterior-lateral and anterior-lateral wedges during marching. Also, the peak of plantar pressure and impulse in the first to third metatarsal regions decreased in the anterior, 
medial and anterior-medial wedges compared to the lateral and posterior-lateral wedges. It seems that using anterior, medial and anterior-medial wedge for insole may have some advantage through increasing comfort sense and decreasing the risk of stress fracture injuries in the second and third metatarsal regions. These findings should be considered during designation of shoes or insoles for military environments.

In addition, a significant negative relation between the comfort level and plantar pressure measurements was observed in the second and third metatarsals and midfoot regions during marching. It seems that comfort assessment may be useful and used instead of plantar pressure measurements when specialized tools is not existed.

\section{Limitations}

The limitations of present study including no using professional soldiers or clinical treatment groups are suggested to future studies to assess more precisely other outcomes or clinical relevance in different populations following use of various insole wedges.

\section{Declarations}

\section{Declaration of Competing Interest}

The authors declare no conflict of interest

\section{References}

1. A. Muniz and R. Bini, "Shock attenuation characteristics of three different military boots during gait," Gait Posture, vol. 58, pp. 59-65, 2017, doi: 10.1016/j.gaitpost.2017.07.037.

2. C. M. House, S. J. Dixon, and A. J. Allsopp, "User Trial and Insulation Tests to Determine Whether Shock- Absorbing Insoles Are Suitable for Use by Military Recruits during Training," Mil. Med., vol. 169, no. 9, pp. 741-746, 2004.

3. P. Paisis, B. Hanley, K. Havenetidis, and A. Bissas, "Cypriot and Greek Army Military Boot Cushioning: Ground Reaction Forces and Subjective Responses," Mil. Med., vol. 178, pp. 493-498, 2013.

4. A. Arndt, P. Westblad, I. Ekenman, and A. Lundberg, "A comparison of external plantar loading and in vivo local metatarsal deformation wearing two different military boots," Gait Posture, vol. 18, pp. 2026, 2003.

5. C. M. Windle, S. M. Gregory, and S. J. Dixon, "The shock attenuation characteristics of four different insoles when worn in a military boot during running and marching," Gait Posture, vol. 9, pp. 31-37, 1999.

6. M. Anbarian and H. Esmaeili, "Effects of running-induced fatigue on plantar pressure distribution in novice runners with different foot types," Gait Posture, vol. 48, pp. 52-56, 2016. 
7. T. M. Willems, R. De Ridder, and P. Roosen, "The effect of a long-distance run on plantar pressure distribution during running," Gait Posture, vol. 35, no. 3, pp. 405-409, 2012.

8. R. Weist, E. Eils, and D. Rosenbaum, "The influence of muscle fatigue on electromyogram and plantar pressure patterns as an explanation for the incidence of metatarsal stress fractures," Am. J. Sports Med., vol. 328, pp. 1893-1898, 2004.

9. M. Nunns, S. J. Dixon, J. Clarke, and M. Carré, "Boot-insole effects on comfort and plantar loading at the heel and fifth metatarsal during running and turning in soccer," J. Sports Sci., 2015.

10. L. Yung-Hui and H. Wei-Hsien, "Effects of shoe inserts and heel height on foot pressure, impact force, and perceived comfort during walking," Appl. Ergon., vol. 36, no. 3, pp. 355-362, 2005.

11. J. Lange, C. Maiwald, T. Mayer, S. Schwanitz, S. Odenwald, and T. Milani, "Relationship between plantar pressure and perceived comfort in military boots," Footwear Sci., vol. 1, no. S1, pp. 30-32, 2009, doi: 10.1080/19424280902977129.

12. H. Chen, B. Nigg, and J. de Koning, "Relationship between plantar pressure distribution under the foot and insole comfort," Clin Biomech, vol. 9, pp. 335-341, 1994.

13. B. . Nigg, J. Baltich, S. Hoerzer, and H. Enders, "Running shoes and running injuries: mythbusting and a proposal for two new paradigms: 'preferred movement path' and 'comfort filter'," Br J Sport. Med, vol. 0, pp. 1-6, 2015, doi: 10.1136/bjsports-2015-095054.

14. F. Yang, Z. Liu, R. Ma, and W. Lam, "Using plantar pressure to predict insole comfort in running," Footwear Sci., vol. 4280, 2019.

15. D. Williams and I. McClay, "Measurements used to characterize the foot and the medial longitudinal arch: reliability and validity," Phys Ther, vol. 80, no. 9, pp. 864-871, 2000, doi: 10.1093/ptj/80.9.864.

16. M. H. Ghasemi, M. Anbarian, and H. Esmaeili, "Effect of various foot wedge conditions on the electromyographic activity of lower extremity muscles during load lifting," Hum Factors Erg. Man, vol. 28 , no. 4, pp. 213-219, 2018, doi: 10.1002/hfm.20736.

17. G. S. Murley, K. B. Landorf, and H. B. Menz, "Do foot orthoses change lower limb muscle activity in flat-arched feet towards a pattern observed in normal-arched feet?," Clin. Biomech., vol. 25, no. 7, pp. 728-736, 2010, doi: 10.1016/j.clinbiomech.2010.05.001.

18. M. Salavati et al., "Test-retest reliabty of center of pressure measures of postural stability during quiet standing in a group with musculoskeletal disorders consisting of low back pain, anterior cruciate ligament injury and functional ankle instability," Gait Posture, vol. 29, no. 3, pp. 460-464, 2009, doi: 10.1016/j.gaitpost.2008.11.016.

19. R. A. Armstrong, "When to use the Bonferroni correction," Ophthalmic \& physiological optics: the journal of the British College of Ophthalmic Opticians (Optometrists), vol. 34, no. 5. pp. 502-508, 01Sep-2014, doi: 10.1111/opo.12131.

20. A. Mundermann, D. J. Stefanyshyn, and B. M. Nigg, "Relationship between footwear comfort of shoe inserts and anthropometric and sensory factors," Med. Sci. Sports Exerc., vol. 33, no. 11, pp. 19391945, 2001. 
21. R. Xu et al., "Comparative Study of the Effects of Customized 3D printed insole and Prefabricated Insole on Plantar Pressure and Comfort in Patients with Symptomatic Flatfoot," Med. Sci. Monit. Int. Med. J. Exp. Clin. Res., vol. 25, pp. 3510-3519, 2019.

\section{Figures}
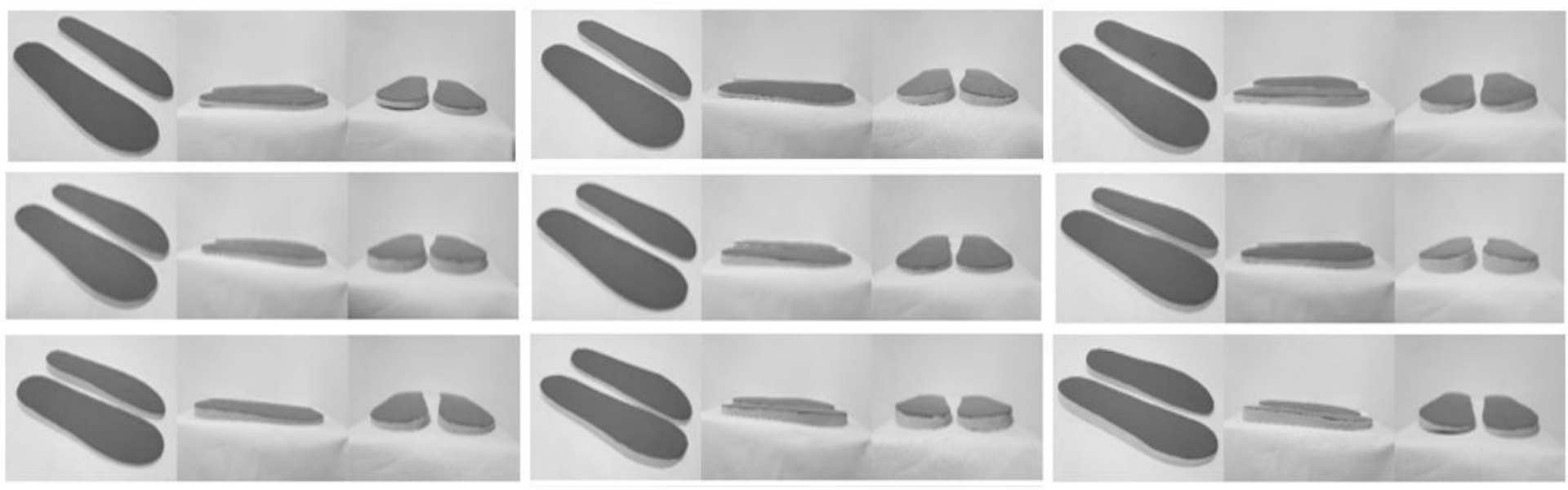

\section{Figure 1}

Insoles designed with various wedges from front, side and diagonal views.

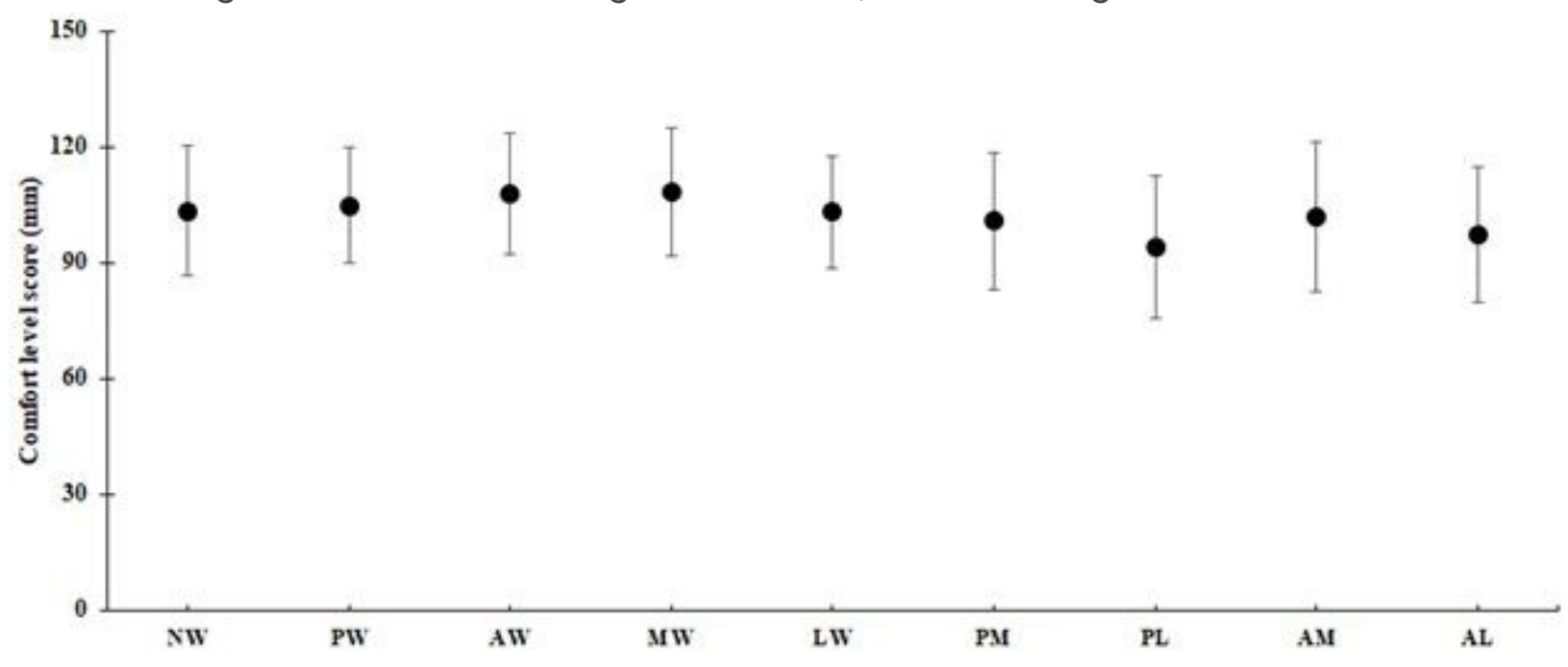

Figure 2

Comparison of mean (SD) of comfort level at various insole wedges during marching. 